\title{
Multiparameter Fractional Difference Linear Control Systems
}

\author{
Dorota Mozyrska \\ Faculty of Computer Science, Bialystok University of Technology, Wiejska 45A, 15-351 Bialystok, Poland \\ Correspondence should be addressed to Dorota Mozyrska; d.mozyrska@pb.edu.pl \\ Received 3 November 2013; Revised 2 January 2014; Accepted 7 January 2014; Published 2 March 2014 \\ Academic Editor: Garyfalos Papashinopoulos
}

Copyright (C) 2014 Dorota Mozyrska. This is an open access article distributed under the Creative Commons Attribution License, which permits unrestricted use, distribution, and reproduction in any medium, provided the original work is properly cited.

\begin{abstract}
The Riemann-Liouville-, Caputo-, and Grünwald-Letnikov-type fractional order difference operators are discussed and used to state and solve the controllability and observability problems of linear fractional order discrete-time control systems with multiorder and multistep. It is shown that the obtained results do not depend on the type of fractional operators and steps. The comparison of systems is made under the number of steps needed, firstly to achieve a final point, and secondly to distinguish initial conditions for particular operator.
\end{abstract}

\section{Introduction}

The main topic of the paper concerns systems with fractional difference operators. Fractional sums and operators are generalizations of $n$th order differences. Since discrete dynamics are often used in modeling real phenomena and recently there is noticed great progress in using fractional dynamics for descriptions of some behaviours, the discussion of properties of possible discrete fractional control systems is strongly needed. Particularly, we consider systems with multiorder and multistep, which could help better in an attempt at simulation of processes and could be easily adapted by CAS programs. Properties of the fractional sums and operators were developed firstly by Miller and Ross [1] and continued by Atici and Eloe $[2,3]$ and Abdeljawad et al. $[4,5]$. Another concept of the fractional sum/difference was introduced in [6-8]. We use in the paper sums and operators with $h$-differences. Since $h>0$ can represent a sample step, the presence of $h$ in operators is interesting from both engineering and numerical points of view. We analyze the controllability and observability problem for multiorder and multistep linear systems while the problems of local controllability of nonlinear control systems defined by Caputotype, Riemann-Liouville-type, and Grünwald-Letnikov-type difference operators, with step $h=1$ and commensurate order, were studied in [9]. Relations between these three types of operators were studied in [10].
In this contribution, the Riemann-Liouville-, Caputo-, and Grünwald-Letnikov-type fractional order difference operators are discussed and used to state and solve the controllability and observability problems of linear fractional order discrete-time control systems with multiorder and multistep. Our goal is to state basic properties of fractional difference multiorder and multistep linear control systems and to compare using some particular examples if there are some varieties between operators and numbers of steps that we need to have a system being controllable or observable or achieve some exact target. It is shown that the obtained results do not depend on the type of fractional operators and steps. The comparison of systems is made under the number of steps needed, firstly to achieve a final point and secondly to distinguish initial conditions for particular operator. As our results are formulated for multistep systems, they remain also true for systems with the same step along coordinates of solutions and particularly for the common step $h=1$. We can also conclude similar corollaries comparing systems according to different or the same orders.

In the paper definitions of operators for general step and orders are presented. In our systems we use $n$ different orders and steps, so we define the multiorder as $(\alpha)=\left(\alpha_{1}, \ldots, \alpha_{n}\right)$, where $\alpha_{i} \in(0,1]$ and multistep $(h)=\left(h_{1}, \ldots, h_{n}\right), h_{i}>0$ for each $i=1, \ldots, n$. In our paper we give proofs for solutions and some properties of systems defined by Riemann-Liouvilletype fractional difference operator and using the formula of 
transformations between Riemann-Liouville and GrünwaldLetnikov types of operators presented in [10], we state results also for systems with Grünwald-Letnikov-type operator.

The paper is organized as follows. In Section 2, all preliminary definitions, facts, and notations are gathered. Section 3 presents initial value problems of fractional order systems of multiorder and multistep difference equations with three types of operators. There are considered systems with operators written in general form. In Section 4, the controllability problem for generalized form of systems is stated and solved, while in Section 5 the observability issue is discussed. At the end of Section 5 there is the example of flat fractional system. The problem of observability of this system is discussed.

\section{Preliminaries}

Now we are listing the necessary definitions and technical propositions that we use in the sequel of the paper. Let $h>0$, $a \in \mathbb{R}$ and put

$$
(h \mathbb{N})_{a}:=\{a, a+h, a+2 h, \ldots\} .
$$

Then $\mathbb{N}_{0}=\{0,1,2, \ldots\}$. For a function $x:(h \mathbb{N})_{a} \rightarrow \mathbb{R}$ the forward $h$-difference operator is defined as (see [7])

$$
\left(\Delta_{h} x\right)(t)=\frac{x(\sigma(t))-x(t)}{h}
$$

where $t \in(h \mathbb{N})_{a}$. In many papers there are defined fractional summations using the special kind of function called factorial function; see, for example, $[7,8,10]$. In order to have here simpler notation we use in definition generalized binomial function $\left(\begin{array}{l}\alpha \\ \beta\end{array}\right)=\Gamma(\alpha+1) / \Gamma(\beta+1) \Gamma(\alpha-\beta+1)$, where $\alpha, \beta \in$ $\mathbb{R} \backslash\{-1,-2, \ldots\}$ and $\Gamma$ is the Euler gamma function. We use the convention that division at a pole yields zero.

Definition 1. For a function $x:(h \mathbb{N})_{a} \rightarrow \mathbb{R}$ the fractional $h$-sum of order $\alpha>0$ is given by

$$
\left({ }_{a} \Delta_{h}^{-\alpha} x\right)(t):=h^{\alpha} \sum_{s=0}^{q}\left(\begin{array}{c}
q-s+\alpha-1 \\
q-s
\end{array}\right) x(a+s h)
$$

where $t=a+(\alpha+q) h, q \in \mathbb{N}_{0}$, and additionally $\left({ }_{a} \Delta_{h}^{0} x\right)(t):=$ $x(t)$.

For $a=0$ we write shortly $\Delta_{h}^{-\alpha}$ instead of ${ }_{0} \Delta_{h}^{-\alpha}$. Note that ${ }_{a} \Delta_{h}^{-\alpha} x:(h \mathbb{N})_{a+\alpha h} \rightarrow \mathbb{R}$. Two fractional $h$-sums can be composed as follows.

Proposition 2 (see [11]). Let $x$ be a real valued function defined on $(h \mathbb{N})_{a}$, where $a, h>0$. For $\alpha, \beta>0$ the following equalities hold:

$$
\begin{aligned}
\left({ }_{a+\beta h} \Delta_{h}^{-\alpha}\left({ }_{a} \Delta_{h}^{-\beta} x\right)\right)(t) & =\left({ }_{a} \Delta_{h}^{-(\alpha+\beta)} x\right)(t) \\
& =\left({ }_{a+\alpha h} \Delta_{h}^{-\beta}\left({ }_{a} \Delta_{h}^{-\alpha} x\right)\right)(t),
\end{aligned}
$$

where $t \in(h \mathbb{N})_{a+(\alpha+\beta) h}$.
In [7] the authors prove the following lemma that gives transition between fractional summation operators for any $h>0$ and $h=1$.

Lemma 3. Let $x:(h \mathbb{N})_{a} \rightarrow \mathbb{R}$ and $\alpha>0$. Then,

$$
\left({ }_{a} \Delta_{h}^{-\alpha} x\right)(t)=h^{\alpha}\left({ }_{a / h} \Delta_{1}^{-\alpha} \tilde{x}\right)\left(\frac{t}{h}\right)
$$

where $t \in(h \mathbb{N})_{a+\alpha h}$ and $\tilde{x}(s)=x(s h)$.

In our consideration the crucial role is played by the power rule formula; see [7]:

$$
\left({ }_{a} \Delta_{h}^{-\alpha} \psi\right)(t)=\frac{\Gamma(\mu+1)}{\Gamma(\mu+\alpha+1)}(t-a+\mu h)_{h}^{(\mu+\alpha)},
$$

where $\psi(r)=(r-a+\mu h)_{h}^{(\mu)}, r \in(h \mathbb{N})_{a}$, and $t \in(h \mathbb{N})_{a+\alpha h}$. Then for $\psi \equiv 1$ we have $\mu=0$. Next, taking $t=q h+a+\alpha h$, $q \in \mathbb{N}_{0}$, we get

$$
\begin{aligned}
\left({ }_{a} \Delta_{h}^{-\alpha} 1\right)(t) & =\frac{1}{\Gamma(\alpha+1)}(t-a)_{h}^{(\alpha)}=\frac{\Gamma(q+\alpha+1)}{\Gamma(\alpha+1) \Gamma(q+1)} h^{\alpha} \\
& =\left(\begin{array}{c}
q+\alpha \\
q
\end{array}\right) h^{\alpha} .
\end{aligned}
$$

As the tool in the present consideration we can define the next family of functions. Let

$$
\begin{gathered}
(k):=\left(k_{1}, \ldots, k_{n}\right), \quad k_{i} \in \mathbb{N}_{0}, \\
(\alpha)=\left(\alpha_{1}, \ldots, \alpha_{n}\right), \quad \alpha_{i} \in(0,1] .
\end{gathered}
$$

Moreover, let $\mu=\sum_{i=1}^{n} \alpha_{i} k_{i}$ and $K=\sum_{i=1}^{n} k_{i}$. Then we define the family of functions $\varphi_{(k)}^{*}: \mathbb{Z} \rightarrow \mathbb{R}$ by the following values:

$$
\varphi_{(k)}^{*}(q):= \begin{cases}\left(\begin{array}{c}
q-K+\mu \\
q-K
\end{array}\right), & \text { for } q \in \mathbb{N}_{K} \\
0, & \text { for } q<K .\end{cases}
$$

Note that the following relations hold:

(1) $\varphi_{(0)}^{*}(q)=1, \varphi_{(k)}^{*}(K)=1$, and $\varphi_{e_{i}}^{*}(1)=1$, where $e_{i}$ is the unit vector in $\mathbb{R}^{n}$ with 1 at the $i$ th position and $(0)=(0, \ldots, 0)$ is the zero vector in $\mathbb{R}^{n}$;

(2) $\varphi_{e_{i}}^{*}(q)=\left(\begin{array}{c}q+v_{i} \\ q-1\end{array}\right)=\left(\Delta_{1}^{-\alpha_{i}} 1\right)\left(q+v_{i}\right)$, where $v_{i}=\alpha_{i}-1$;

(3) $\varphi_{(k)}^{*}(q)=\Gamma(q+1-K+\mu) / \Gamma(1+\mu) \Gamma(q+1-K)$ and as the division by pole gives zero, the formula works also for $q<K$;

(4) $\varphi_{(k)}^{*}(q)=(1 / \Gamma(1+\mu))(q-K+\mu)^{(\mu)}=\left(\begin{array}{c}q-K+\mu \\ \mu\end{array}\right)$.

The properties of two-indexed functions $\varphi_{k, s}$ were given in [12, Proposition 2.5] and generalized for $n$-indexed functions $\varphi_{(k)}^{*}$ in $[12,13]$ with multiorder $(\alpha)$. In the paper therein we use functions $\varphi_{(k)}^{*}$ with step $h=1$ which gives better tool for multistep systems. 
Proposition 4. Let for $i=1, \ldots, n: \alpha_{i} \in(0,1]$ and $v_{i}=\alpha_{i}-1$, $q \in \mathbb{N}_{1}, k=\left(k_{1}, \ldots, k_{n}\right) \in \mathbb{R}^{n}$. Then for $q \in \mathbb{N}_{0}$ holds

$$
\left(\Delta^{-\alpha_{i}} \varphi_{(k)}^{*}\right)\left(q+v_{i}\right)=\varphi_{(k)+e_{i}}^{*}(q) .
$$

Family of functions $\varphi_{(k)}^{*}$ is useful for solving systems with Caputo-type operators. We formulate also similar family of functions that are used in solutions of systems with RiemannLiouville-type operators. We define the family of functions $\varphi_{(k)}: \mathbb{Z} \rightarrow \mathbb{R}$ by the following values:

$$
\varphi_{(k)}(q):= \begin{cases}\left(\begin{array}{l}
q-K+\mu \\
q-K+1
\end{array}\right), & \text { for } q \in \mathbb{N}_{K-1} \\
0, & \text { for } q<K-1 .\end{cases}
$$

Note that the following relations hold:

(1) $\varphi_{(k)}(q)=(\mu /(q-K+1)) \varphi_{(k)}^{*}(q)$;

(2) $\varphi_{(0)}(0)=0, \varphi_{(k)}(K)=\mu, \varphi_{e_{i}}(1)=\alpha_{i}$;

(3) for all $(k) \in \mathbb{R}^{n}: \varphi_{(k)}(q) \geq 0$ and $\varphi_{(k)}^{*}(q) \geq 0$.

For the family of functions $\varphi_{(k)}$ we have similar behaviour for fractional summations as for $\varphi_{(k)}^{*}$ so we can state the following proposition.

Proposition 5. Let for $i=1, \ldots, n: \alpha_{i} \in(0,1]$ and $\nu_{i}=\alpha_{i}-1$, $q \in \mathbb{N}_{1}, k=\left(k_{1}, \ldots, k_{n}\right) \in \mathbb{R}^{n}$. Then for $q \in \mathbb{N}_{0}$ holds

$$
\left(\Delta^{-\alpha_{i}} \varphi_{(k)}\right)\left(q+v_{i}\right)=\varphi_{(k)+e_{i}}(q) .
$$

Lemma 6 (see $[12,14])$. Let $u: \mathbb{N}_{0} \rightarrow \mathbb{R}, \alpha>0$. For $k \in \mathbb{N}_{0}$ let $\left(\Delta^{-k \alpha} u\right)(q+k \alpha)=u_{1}(q+k \alpha)$ and $\bar{u}_{1}(q):=u_{1}(q+k \alpha)$, $q \in \mathbb{N}_{0}$. Then for $k \in \mathbb{N}_{0}$ holds

$$
\left(\Delta^{-\alpha} \bar{u}_{1}\right)(t)=\left(\Delta^{-(k+1) \alpha} u\right)(t+k \alpha)
$$

where $t=q+\alpha, q \in \mathbb{N}_{0}$.

Similarly as in [14], taking $h=1$ we can write that for $k_{i} \in \mathbb{N}_{0}$ and $\alpha_{i}>0, i=1, \ldots, n$ :

$$
\left(\Delta^{-\alpha_{j}}\left(\Delta^{-\sum_{i} \alpha_{i} k_{i}} u\right)\right)(t)=\left(\Delta^{-\sum_{i \neq j} \alpha_{i} k_{i}-\left(k_{j}+1\right) \alpha_{j}} u\right)(t),
$$

where $t=q+\sum_{i \neq j} \alpha_{i} k_{i}+\left(k_{j}+1\right) \alpha_{j}, q \in \mathbb{N}_{0}$. Also for $\mu=\sum_{i} \alpha_{i} k_{i}$ and $q \in \mathbb{N}_{0}$ we have

$$
\left(\Delta^{-\mu} u\right)(q+\mu)=\sum_{r=0}^{q}\left(\begin{array}{c}
q-r+\mu-1 \\
q-r
\end{array}\right) u(r) .
$$

The next step is to present three main fractional difference operators: Caputo-type, Riemann-Liouville-type, and Grünwald-Letnikov-type $h$-difference operators. We present all definitions for any positive $h$ and scalar function $x$. In fact each of the operators with step $h>0$ can be translated into the according operator with step $h=1$. We state results about the inversions of operators in two cases for any positive $h$ and $h=1$. Only the case of the Grünwald-Letnikov-type $h$-difference operators has no exact inverse operator, but we can transform an initial value problem stated for this operator into initial value problem for the Riemann-Liouville-type $h$ difference operators, where we change the domain of the state.
Definition 7 (see [11]). Let $\alpha \in(0,1]$. The Caputo-type $h$-difference operator ${ }_{a} \Delta_{h, *}^{\alpha}$ of order $\alpha$ for a function $x$ : $(h \mathbb{N})_{a} \rightarrow \mathbb{R}$ is defined by

$$
\left({ }_{a} \Delta_{h, *}^{\alpha} x\right)(t)=\left({ }_{a} \Delta_{h}^{-(1-\alpha)}\left(\Delta_{h} x\right)\right)(t),
$$

where $t \in(h \mathbb{N})_{a+(1-\alpha) h}$.

Note that ${ }_{a} \Delta_{h, *}^{\alpha} x:(h \mathbb{N})_{a+(1-\alpha) h} \rightarrow \mathbb{R}$. Moreover, for $\alpha=1$ the Caputo-type $h$-difference operator takes the form $\left({ }_{a} \Delta_{h, *}^{1} x\right)(t)=\left({ }_{a} \Delta_{h}^{0}\left(\Delta_{h} x\right)\right)(t)=\left(\Delta_{h} x\right)(t)$, where $t \in(h \mathbb{N})_{a}$.

For the Caputo-type fractional difference operator there exists the inverse operator that is the tool in recurrence and direct solving fractional difference equations.

Proposition 8 (see [11]). Let $\alpha \in(0,1], h>0, a=(\alpha-1) h$, and $x$ be a real valued function defined on $(h \mathbb{N})_{a}$. The following formula holds:

$$
\left(\Delta_{h}^{-\alpha}\left({ }_{a} \Delta_{h, *}^{\alpha} x\right)\right)(t)=x(t)-x(a), \quad t \in(h \mathbb{N})_{\alpha h} .
$$

We can also state, similarly as in Lemma 3, the transition formula for the Caputo-type operator between the cases for any $h>0$ and $h=1$.

Lemma 9. Let $x:(h \mathbb{N})_{a} \rightarrow \mathbb{R}$ and $\alpha>0$. Then,

$$
\left({ }_{a} \Delta_{h, *}^{\alpha} x\right)(t)=h^{-\alpha}\left({ }_{a / h} \Delta_{1, *}^{\alpha} \tilde{x}\right)\left(\frac{t}{h}\right),
$$

where $t \in(h \mathbb{N})_{a+\alpha h}$ and $\widetilde{x}(s)=x(s h)$.

From this moment for the case $h=1$ we write $a_{a / h} \Delta_{*}^{\alpha}:={ }_{a / h} \Delta_{1, *}^{\alpha}$ and the same notation we use for two other operators and fractional summations. Then the result from Proposition 8 is stated as

$$
\left(\Delta^{-\alpha}\left(a / h \Delta_{*}^{\alpha} \tilde{x}\right)\right)(q+\alpha)=\tilde{x}(q+\alpha)-x(a),
$$

where $q \in \mathbb{N}_{0}, \widetilde{x}(s)=x(s h)$, and $a=(\alpha-1) h$.

The next presented operator is called fractional $h$ difference Riemann-Liouville-type operator. The definition of the operator can be found, for example, in [2] (for $h=1)$ or in [6] (for any $h>0$ ).

Definition 10. Let $\alpha \in(0,1]$. The Riemann-Liouville-type fractional $h$-difference operator ${ }_{a} \Delta_{h}^{\alpha} x$ of order $\alpha$ for a function $x:(h \mathbb{N})_{a} \rightarrow \mathbb{R}$ is defined by

$$
\begin{array}{r}
\left({ }_{a} \Delta_{h}^{\alpha} x\right)(t)=\left(\Delta_{h}\left({ }_{a} \Delta_{h}^{-(1-\alpha)} x\right)\right)(t), \\
t \in(h \mathbb{N})_{a+(1-\alpha) h} .
\end{array}
$$

For $\alpha=1$ we have: $\left({ }_{a} \Delta_{h, *}^{1} x\right)(t)=\left({ }_{a} \Delta_{h}^{1} x\right)(t)=\left(\Delta_{h} x\right)(t)$.

The next propositions give useful formula for transforming fractional difference equations into fractional summations. 
Proposition 11. Let $\alpha \in(0,1], h>0, a=(\alpha-1) h$, and $x$ be a real valued function defined on $(h \mathbb{N})_{a}$. The following formula holds:

$$
\left({ }_{0} \Delta_{h}^{-\alpha}\left({ }_{a} \Delta_{h}^{\alpha} x\right)\right)(t)=x(t)-x(a) \cdot\left(\begin{array}{c}
\frac{t}{h} \\
\alpha-1
\end{array}\right), \quad t \in(h \mathbb{N})_{\alpha h} .
$$

The next Lemma gives the transition formula for the Riemann-Liouville-type operator between the cases for any $h>0$ and $h=1$.

Lemma 12. Let $x:(h \mathbb{N})_{a} \rightarrow \mathbb{R}$ and $\alpha>0$. Then,

$$
\left({ }_{a} \Delta_{h}^{\alpha} x\right)(t)=h^{-\alpha}\left({ }_{a / h} \Delta_{1}^{\alpha} \tilde{x}\right)\left(\frac{t}{h}\right),
$$

where $\in(h \mathbb{N})_{a+(1-\alpha) h}$ and $\tilde{x}(s)=x(s h)$.

For the case $h=1$ we write ${ }_{a / h} \Delta^{\alpha}:={ }_{a / h} \Delta_{1}^{\alpha}$. Then the result from Proposition 11 is stated as

$$
\left(\Delta^{-\alpha}\left(a_{h / h} \Delta^{\alpha} \tilde{x}\right)\right)(q+\alpha)=\tilde{x}(q+\alpha)-x(a) \cdot\left(\begin{array}{l}
q+\alpha \\
q+1
\end{array}\right),
$$

where $q \in \mathbb{N}_{0}, \tilde{x}(s)=x(s h)$ and with $a=(\alpha-1) h$.

The third type of the operators that we take into our consideration is the fractional $h$-difference Grünwald-Letnikovtype operator; see, for example, [15-17] for case $h=1$ and also for case $h>0$.

Definition 13. Let $\alpha \in \mathbb{R}$. The Grünwald-Letnikov-type $h$ difference operator ${ }_{a} \widetilde{\Delta}_{h}^{\alpha}$ of order $\alpha$ for a function $x:(h \mathbb{N})_{a} \rightarrow$ $\mathbb{R}$ is defined by

$$
\left({ }_{a} \widetilde{\Delta}_{h}^{\alpha} x\right)(t)=\sum_{s=0}^{(t-a) / h} a_{s}^{(\alpha)} x(t-s h),
$$

where

$$
\begin{aligned}
& a_{s}^{(\alpha)}=(-1)^{s}\left(\begin{array}{l}
\alpha \\
s
\end{array}\right) \frac{1}{h^{\alpha}} \quad \text { with } \\
& \left(\begin{array}{l}
\alpha \\
s
\end{array}\right)= \begin{cases}1 & \text { for } s=0 \\
\frac{\alpha(\alpha-1) \cdots(\alpha-s+1)}{s !} & \text { for } s \in \mathbb{N} .\end{cases}
\end{aligned}
$$

We can easily see the following comparison.

Proposition 14 (see [10]). Let $a=(\alpha-1) h$. Then

$$
\nabla_{h}\left({ }_{a} \Delta_{h}^{-(1-\alpha)} x\right)(n h)=\left({ }_{0} \widetilde{\Delta}_{h}^{\alpha} y\right)(n h),
$$

where $y(n h):=x(n h+a)$ or $x(t)=y(t-a)$ for $t \in(h \mathbb{N})_{a}$ and $\left(\nabla_{h} x\right)(n h)=(x(n h)-x(n h-h)) / h$.

From Proposition 14 we get in the next chapters the possibility of stating exact formula for solution of initial value problems with the Grünwald-Letnikov-type difference operators by the comparison with parallel problems with the Riemann-Liouville-type operators.

The operators presented in this section can be extended to operators acting on vector valued functions in a componentwise manner.

\section{Multiorder and Multistep Fractional Difference Linear Systems}

In this section we consider initial value problems of fractional order systems of multiorder and multistep difference equations with three types of operators. In the next section we state as the conclusion the solution of initial value problem for control systems with the Caputo-, the Riemann-Liouville, and the Grünwald-Letnikov-type difference operators and formulate common results for the controllability as well for observability. We consider systems with operators written in the general form

$$
\left({ }_{a_{i}} Y_{h_{i}}^{\alpha_{i}} x_{i}\right)\left(q h_{i}\right)=\sum_{j=1}^{n} A_{i j} x_{j}\left(q h_{j}+a_{j}\right), \quad q \in \mathbb{N}_{0}
$$

where $i=1, \ldots, n$ and $n \in \mathbb{N}_{1}$, with the initial condition

$$
x_{(a)}=\left[\begin{array}{c}
x_{1}\left(a_{1}\right) \\
\vdots \\
x_{n}\left(a_{n}\right)
\end{array}\right]=\left[\begin{array}{c}
x_{a_{1}} \\
\vdots \\
x_{a_{n}}
\end{array}\right] \in \mathbb{R}^{n} \text {. }
$$

Therein functions $x_{i}:(h \mathbb{N})_{a_{i}} \rightarrow \mathbb{R}, A_{i j} \in \mathbb{R}, \bar{x}_{i}(s)=\widetilde{x}_{i}(s+$ $\left.v_{i}\right)=x_{i}\left(a_{i}+s h_{i}\right), a_{i}=v_{i} h_{i}=\left(\alpha_{i}-1\right) h_{i}$ if ${ }_{a_{i}} \Upsilon_{h_{i}}^{\alpha_{i}}={ }_{a_{i}} \Delta_{h_{i}, *}^{\alpha_{i}}$ or $a_{i} \Delta_{h_{i}}^{\alpha_{i}}$ and $a_{i}=0$ if ${ }_{a_{i}} Y_{h_{i}}^{\alpha_{i}}\left(q h_{i}\right)={ }_{0} \widetilde{\Delta}_{h_{i}}^{\alpha_{i}}\left(q h_{i}+h_{i}\right)$. We stress that for the Grünwald-Letnikov-type the left side of (27) is shifted; compare [10]. By Lemma 9 we can write system (27) in the form

$$
\begin{gathered}
\left({ }_{v_{i}} \Upsilon^{\alpha_{i}} \tilde{x}_{i}\right)(q)=h_{i}^{\alpha_{i}} \sum_{j=1}^{n} A_{i j} \bar{x}_{j}(q), \quad i=1, \ldots, n, \\
\bar{x}_{i}(0)=x_{a_{i}},
\end{gathered}
$$

where $v_{i}=a_{i} / h_{i}=\alpha_{i}-1$.

Theorem 15. The solution of system (27) with initial condition (28) is of the form

$$
\left[\begin{array}{c}
x_{1}\left(q h_{1}+a_{1}\right) \\
\vdots \\
x_{n}\left(q h_{n}+a_{n}\right)
\end{array}\right]=\Phi(q)\left[\begin{array}{c}
x_{a_{1}} \\
\vdots \\
x_{a_{n}}
\end{array}\right]
$$

where for $q \in \mathbb{N}_{0}$ :

$$
\Phi(q)=\sum_{(k): 0 \leq \sum_{i=1}^{n} k_{i} \leq q} T_{(k)} M_{(k)}(q),
$$




$$
\begin{aligned}
& M_{k}(q) \\
& = \begin{cases}\varphi_{(k)}^{*}(q) I_{n}, & { }_{a_{i}} \Upsilon_{h_{i}}^{\alpha_{i}}={ }_{a_{i}} \Delta_{h_{i}, *}^{\alpha_{i}} ; \\
\operatorname{diag}\left(\varphi_{e_{1}+(k)}, \varphi_{e_{2}+(k)}, \ldots, \varphi_{e_{n}+(k)}\right)(q), & { }_{a_{i}} \Upsilon_{h_{i}}^{\alpha_{i}}={ }_{a_{i}} \Delta_{h_{i}}^{\alpha_{i}},\end{cases}
\end{aligned}
$$

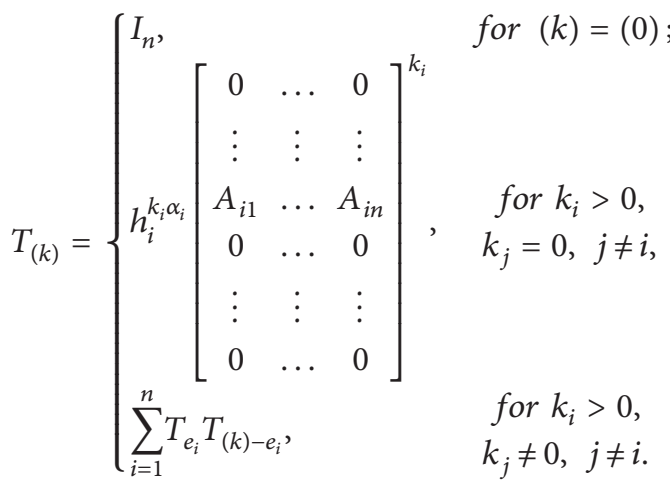

Proof. The most important step in the proof is to use the properties of fractional summations with different orders of functions from families $\varphi_{(k)}^{*}$ and $\varphi_{(k)}$. Let $x_{(a)}=\left[\begin{array}{c}x_{a_{1}} \\ \vdots \\ x_{a_{n}}\end{array}\right]$ and $v_{i}=a_{i} / h_{i}=\alpha_{i}-1$. For $q=0$ we see that $\Phi(0)=T_{(0)} M_{(0)}(0)=$ $I_{n}$; hence, the formula agrees with the initial condition. The only one doubt that we should check is the value of $M_{(0)}(0)$ for the case of Riemann-Liouville-type operator. In fact we have $M_{(0)}(0)=\operatorname{diag}\left(\varphi_{e_{1}}(0), \ldots, \varphi_{e_{n}}(0)\right)=I_{n}$. By Proposition 8 we obtain the recursive formulas for the Caputo-type operator, $i=1, \ldots, n$ and $q \in \mathbb{N}_{1}, v_{i}=\alpha_{i}-1$ :

$$
x_{i}\left(q h_{i}+a_{i}\right)=\bar{x}_{i}(q)=x_{a_{i}}+h_{i}^{\alpha_{i}} \sum_{j=1}^{n} A_{i j}\left(\Delta^{-\alpha_{i}} \bar{x}_{j}\right)\left(q+v_{i}\right) .
$$

Since for the Riemann-Liouville-type operator we start with $\bar{x}_{i}(q)=x_{a_{i}} \varphi_{e_{i}}(q)=x_{a_{i}}\left(\begin{array}{c}q+\alpha_{i}-1 \\ q\end{array}\right)$, from Proposition 11 we obtain

$$
\begin{aligned}
x_{i}\left(q h_{i}+a_{i}\right)= & \bar{x}_{i}(q)=\varphi_{e_{i}}(q) x_{a_{i}} \\
& +h_{i}^{\alpha_{i}} \sum_{j=1}^{n} A_{i j}\left(\Delta^{-\alpha_{i}} \bar{x}_{j}\right)\left(q+v_{i}\right) .
\end{aligned}
$$

Using matrices $T_{(0)}, T_{e_{i}}$ and functions $M_{(k)}(q)$, recursive relations (33) and (34) could be rewritten in the form

$$
\bar{x}(q)=T_{(0)} M_{(0)}(q) x_{(a)}+\sum_{i=1}^{n} T_{e_{i}}\left(\Delta^{-\alpha_{i}} \bar{x}\right)\left(q+v_{i}\right)
$$

or equivalently as

$$
\begin{aligned}
\bar{x}(q)= & {\left[\begin{array}{c}
\bar{x}_{1} \\
\vdots \\
\bar{x}_{n}
\end{array}\right](q) } \\
= & T_{(0)} M_{(0)}(q)\left[\begin{array}{c}
x_{a_{1}} \\
\vdots \\
x_{a_{n}}
\end{array}\right] \\
& +\sum_{i=1}^{n} T_{e_{i}}\left(\Delta^{-\alpha_{i}}\left[\begin{array}{c}
\bar{x}_{1} \\
\vdots \\
\bar{x}_{n}
\end{array}\right]\right)\left(q+v_{i}\right) .
\end{aligned}
$$

It is important to stress that the recursive formula (36) and the initial value problem given by the system (27) with initial condition (28) are equivalent. Moreover, the recursive formula (36) represents the unique solution to the initial value problem. In order to prove that formula (30) is the solution to the stated problem we can check that it satisfies the equality (36) and initial condition. The zero step for initial condition has been already checked. Then, the crucial role is played by the following property:

$$
\left(\Delta^{-\alpha_{i}} M_{(k)}\right)\left(q+v_{i}\right)=M_{(k)+e_{i}}(q),
$$

that follows from Propositions 4 and 5. Moreover, as for sequences $(k)$ such that $\sum_{i=1}^{n} k_{i}>q$ we have $M_{(k)}(q)=0$; then the values of the matrix $\Phi$ in the formula (31) can be written as an infinite summation:

$$
\begin{aligned}
\Phi(q) & =\sum_{(k): 0 \leq \sum_{i=1}^{n} k_{i} \leq q} T_{(k)} M_{(k)}(q) \\
& =\sum_{(k): \sum_{i=1}^{n} k_{i} \geq 0} T_{(k)} M_{(k)}(q) .
\end{aligned}
$$

Let $K=\sum_{i=1}^{n} k_{i}$. Then the right-hand side of the recursive formula (36) takes the form

$$
\begin{aligned}
T_{(0)} & M_{(0)}(q) x_{(a)}+\sum_{i=1}^{n} T_{e_{i}}\left(\Delta^{-\alpha_{i}} \bar{x}\right)\left(q+v_{i}\right) \\
& =\left(T_{(0)} M_{(0)}(q)+\sum_{i=1}^{n} T_{e_{i}} \sum_{(k): K \geq 0} T_{(k)} M_{(k)+e_{i}}(q)\right) x_{(a)} \\
& =\left(T_{(0)} M_{(0)}(q)+\sum_{i=1}^{n} T_{e_{i}} \sum_{(k): K \geq 1} T_{(k)-e_{i}} M_{(k)}(q)\right) x_{(a)} \\
& =T_{(0)} M_{(0)}(q) x_{(a)}+\sum_{(k): K \geq 1} \sum_{\sum_{i=1}^{n} T_{e_{i}} T_{(k)-e_{i}}} M_{(k)}(q) x_{(a)} \\
= & \sum_{(k): K \geq 0} T_{(k)} M_{(k)}(q) x_{(a)} \\
= & \sum_{(k): 0 \leq K \leq q} T_{(k)} M_{(k)}(q) x_{(a)}=\bar{x}(q) .
\end{aligned}
$$

Hence the thesis holds. 


\section{Controllability}

In this section we construct the solution of initial value problems and consider the controllability problem of multistep and multiorder difference linear control systems for different forms of operators. form

We consider systems with operators written in the general

$$
\left(a_{i} \Upsilon_{h_{i}}^{\alpha_{i}} x_{i}\right)\left(q h_{i}\right)=\sum_{j=1}^{n} A_{i j} x_{j}\left(q h_{j}+a_{j}\right)+b_{i} u(q),
$$

$$
q \in \mathbb{N}_{0}
$$

where $i=1, \ldots, n$ and $n \in \mathbb{N}_{1}$ and with initial condition

$$
x_{(a)}=\left[\begin{array}{c}
x_{1}\left(a_{1}\right) \\
\vdots \\
x_{n}\left(a_{n}\right)
\end{array}\right]=\left[\begin{array}{c}
x_{a_{1}} \\
\vdots \\
x_{a_{n}}
\end{array}\right] \in \mathbb{R}^{n} .
$$

Therein functions $x_{i}:(h \mathbb{N})_{a_{i}} \rightarrow \mathbb{R}, A_{i j} \in \mathbb{R}, \bar{x}_{i}(s)=\tilde{x}_{i}(s+$ $\left.v_{i}\right)=x\left(s h_{i}+a_{i}\right)$ with $a_{i}=\left(\alpha_{i}-1\right) h_{i}$ and $v_{i}=\alpha_{i}-1$ if ${ }_{a_{i}} Y_{h_{i}}^{\alpha_{i}}=$ ${ }_{a_{i}} \Delta_{h_{i}, *}^{\alpha_{i}}$ or ${ }_{a_{i}} \Delta_{h_{i}}^{\alpha_{i}}$ and $v_{i}=0$ if ${ }_{a_{i}} \Upsilon_{h_{i}}^{\alpha_{i}}={ }_{0} \widetilde{\Delta}_{h_{i}}^{\alpha_{i}}$. Moreover, $b_{i} \epsilon$ $\mathbb{R}^{1 \times m}$ are constant-row matrices. By Lemmas 9 and 12 we can write system (40) in the form

$$
\begin{array}{r}
\left({ }_{v_{i}} \Upsilon^{\alpha_{i}} \tilde{x}_{i}\right)(q)=h_{i}^{\alpha_{i}} \sum_{j=1}^{n} A_{i j} \bar{x}_{j}(q)+h_{i}^{\alpha_{i}} b_{i} u(q), \\
i=1, \ldots, n, \quad q \in \mathbb{N}_{0} .
\end{array}
$$

Theorem 16. Let

$$
B_{i}=\left[\begin{array}{c}
0 \\
b_{i} h_{i}^{\alpha_{i}} \\
0
\end{array}\right] \in \mathbb{R}^{n \times m}
$$

be the ith row which is equal to $b_{i} h_{i}^{\alpha_{i}}$. Then, the solution of system (42) with the initial condition $x_{(a)}=x_{a} \in \mathbb{R}^{n}$ which is unique and given by

$$
\gamma\left(q, x_{(a)}, u\right)=\Phi(q) x_{(a)}+\sum_{i=1}^{n} \sum_{r=0}^{q} E_{i}(q-\sigma(r)) B_{i} u(r),
$$

where $q \in \mathbb{N}_{0}$ and

$$
\begin{gathered}
E_{i}(q)=\sum_{(k): 0 \leq \sum_{i}^{n} k_{i} \leq q} T_{(k)} \widetilde{\varphi}_{(k)+e_{i}}(q), \\
\widetilde{\varphi}_{(k)}(q):=\left(\begin{array}{c}
q+\mu-1 \\
q
\end{array}\right), \quad \mu=\sum_{i=1}^{n} k_{i} \alpha_{i} .
\end{gathered}
$$

Proof. The steps in the proof are similar to the case with one value for the step $h=1$ like it is presented in [14].

We can consider also the value $E(-1)=0$. Then for $q \in \mathbb{N}_{1}$ the solution of (40) can be rewritten in the form

$$
\gamma\left(q, x_{(a)}, u\right)=\Phi(q) x_{(a)}+\sum_{i=1}^{n} \sum_{r=0}^{q-1} E_{i}(q-\sigma(r)) B_{i} u(r) .
$$

Definition 17. System (40) is (completely) controllable in qsteps from the initial state (41) to the final state

$$
x_{(f)}=\left[\begin{array}{c}
x_{f_{1}} \\
\vdots \\
x_{f_{n}}
\end{array}\right] \in \mathbb{R}^{n}
$$

such that $x_{(f)} \neq x_{(a)}$, if there is a control $u$, such that

$$
\gamma\left(q, x_{(a)}, u\right)=x_{(f)} .
$$

Note that controllability means that the final state can be reached in $q$-steps, where $q \in \mathbb{N}_{1}$.

Let us define the controllability matrix for the system (42) in the following way, as in [13]:

$$
Q_{q}=\sum_{r=0}^{q-1} P(q, r) P^{T}(q, r)
$$

where

$$
P(q, r)=\sum_{i=1}^{n} E_{i}(q-\sigma(r)) B_{i}
$$

and $P^{T}(q, r)$ denotes the transposition of the matrix $P(q, r)$. It can be noticed that $Q_{q}$ is a symmetric $n \times n$-dimensional matrix.

The next propositions give the formula for the values of steering control and also rank condition for complete controllability. The results have the same meaning like in the classical theory. For the fractional difference systems with the Caputo-type operator with one step $h$ and two orders $(\alpha)=\left(\alpha_{1}, \alpha_{2}\right)$ some conditions were proved in [13]. The basic difference here is the form of the matrices $T_{(k)}$ and $B_{i}$, where we have multiplication of rows by $h_{i}^{k_{i} \alpha_{i}}$ or only $h_{i}^{\alpha_{i}}$ for $b_{i}$, which can influence the rank of controllability matrix $Q_{q}$. See the example in the end of the next section. It is important to notice that the form of the controllability matrix and of the formula of values $\bar{u}(r)>0$ do not depend on the type of the operator.

Proposition 18 (see [13]). If the matrix $Q_{q}$ is nonsingular, then the following control function

$$
\bar{u}(r)=P^{T}(q, r) \cdot Q_{q}^{-1}\left(x_{(f)}-\Phi(q) x_{(a)}\right)
$$

transfers the initial state $x_{(a)}$ to the final state $x_{(f)}=\gamma\left(q, x_{(a)}\right.$, $u)$.

Proposition 19. Control system (40) is controllable in q steps if and only if rank $Q_{q}=n$.

\section{Observability}

Let us consider multiorder and multistep difference linear control system of the form (40), written as a common form for each operator, where the output function is the same

$$
\eta(q)=C \bar{x}(q)+D u(q)
$$


as initial condition $x_{(a)}$ given by (41), where $q \in \mathbb{N}_{0}$. Note that for $q \in \mathbb{N}_{0}$ from the output relation (52) it follows that the output trajectory of this system, denoted by $\chi\left(\cdot, x_{(a)}, u\right)$, is given by

$$
\begin{aligned}
& \chi\left(q, x_{(a)}, u\right) \\
&= C \Phi(q) x_{(a)}+C \cdot \sum_{i=1}^{n} \sum_{r=0}^{q} E_{i}(q-\sigma(r)) B_{i} u(r) \\
&+D u(q),
\end{aligned}
$$

where $x_{(a)}$ is the initial state described by $(41)$ and $E(-1)=0$.

Definition 20. System (40) with the output function (52) is observable in $q$ steps, if having a control $u=$ $\{u(0), u(1), \ldots, u(q)\}$, and output sequence $y=\{\eta(0), \eta(1)$, $\ldots, \eta(q)\}$ one can determinate the initial condition $x_{(a)}$ of this system.

Let us define a real matrix

$$
\mathcal{O}_{q}=\sum_{r=0}^{q} \Phi^{T}(r) C^{T} C \Phi(r)
$$

where $q \in \mathbb{N}_{0}$ and $\Phi^{T}$ denotes the transposition of $\Phi$. Since it is defined in the similar manner as the classical observability Gramian, we call it the (fractional) observability Gramian. Observe that $C \in \mathbb{R}^{p \times n}$ and so $C \Phi(q) \in \mathbb{R}^{p \times n}$.

Theorem 21. System (40) with the output function (52) is observable in $q$ steps if and only if matrix $\mathcal{O}_{q}$ given by (54) is nonsingular.

At the end we give the example in which we show that for a given system with the Riemann-Liouville-type operator we have faster recognition of the initial state than for the system with the Caputo-type operator.

Theorem 22. System (40) with the output (52) is observable in q steps if and only if one of the following conditions is satisfied:

(i) columns of the matrix $C \Phi(q)$ are linearly independent;

(ii) $\operatorname{rank}\left[\begin{array}{c}C \Phi(0) \\ \vdots \\ C \Phi(q)\end{array}\right]=n$.

The matrix

$$
\left[\begin{array}{c}
C \Phi(0) \\
\vdots \\
C \Phi(q)
\end{array}\right]
$$

is called the (fractional) observability matrix. Directly from the fact that matrix $\mathcal{O}_{q}$ is nonsingular and from the proof of Theorem 21 it follows that this matrix is positively defined.
Example 23. Let us consider linear control system with two fractional orders $\alpha \neq \beta$ and with two steps $(h)=\left(h_{1}, h_{2}\right)$ :

$$
\begin{gathered}
\left(a_{1} \Upsilon_{h_{1}}^{\alpha} \bar{x}\right)(q)=\bar{x}(q)+2 \tilde{y}(q), \\
\left(a_{2} \Upsilon_{h_{2}}^{\beta} \bar{y}\right)(q)=2 \bar{x}(q)+\tilde{y}(q), \\
\eta(q)=\bar{x}(q)+\tilde{y}(q)
\end{gathered}
$$

with initial condition $\left[x\left(a_{1}\right), y\left(a_{2}\right)\right]=[\bar{x}(0), \tilde{y}(0)]$. Let $x$ : $(h \mathbb{N})_{a} \rightarrow \mathbb{R}, y:(h \mathbb{N})_{b} \rightarrow \mathbb{R}$

(1) Firstly we consider the situation with the Caputo-type difference and $h_{1}=h_{2}=1$. Note that $T_{(0,0)}=I_{2}$ and $\varphi_{(0)}^{*}(q)=1, \varphi_{e_{i}}^{*}(1)=1$. Then $\Phi(1)=T_{(0)} \varphi_{(0)}^{*}(1)+$ $T_{(0,1)} \varphi_{(1,0)}^{*}(1)+T_{(0,1)} \varphi_{(0,1)}^{*}(1)=\left[\begin{array}{ll}2 & 2 \\ 2 & 2\end{array}\right]$. This implies that

$$
\operatorname{rank}\left[\begin{array}{l}
C \Phi(0) \\
C \Phi(1)
\end{array}\right]=1,
$$

so our system is not observable in $q=1$ step. For $q=2$ we have

$$
\begin{gathered}
\varphi_{(1,0)}^{*}(2)=1+\alpha, \quad \varphi_{(0,1)}^{*}(2)=1+\beta, \\
\varphi_{(1,1)}^{*}(2)=\varphi_{(2,0)}^{*}(2)=\varphi_{(0,2)}^{*}(2)=1, \\
T_{(1,1)}=\left[\begin{array}{ll}
4 & 2 \\
2 & 4
\end{array}\right], \quad T_{(2,0)}=\left[\begin{array}{ll}
1 & 2 \\
0 & 0
\end{array}\right], \quad T_{(0,2)}=\left[\begin{array}{ll}
0 & 0 \\
2 & 1
\end{array}\right] .
\end{gathered}
$$

Now we are ready to state the value of fundamental matrix for $q=2: \Phi(2)=T_{(0)} \varphi_{(0)}^{*}(2)+T_{(0,1)} \varphi_{(1,0)}^{*}(2)+$ $T_{(0,1)} \varphi_{(0,1)}^{*}(2)+2 T_{(1,1)} \varphi_{(1,1)}^{*}(2)+T_{(2,0)} \varphi_{(2,0)}^{*}(2)+$ $T_{(0,2)} \varphi_{(0,2)}^{*}(2)=\left[\begin{array}{ll}11+\alpha & 8+2 \alpha \\ 8+2 \beta & 11+\beta\end{array}\right]$. Then $C \Phi(2)=[19+\alpha+$ $2 \beta, 19+2 \alpha+\beta]$. Hence for $\alpha \neq \beta$ holds rank $\left[\begin{array}{c}C \Phi(0) \\ C \Phi(1) \\ C \Phi(2)\end{array}\right]=$ 2 , so the system is observable in $q=2$ step.

(2) Taking into account the Caputo-type operator and $h_{1}=1, h_{2}=h \neq 1$ we get $\Phi(1)=I_{2}+\left[\begin{array}{ll}1 & 2 \\ 0 & 0\end{array}\right]+h^{\beta}\left[\begin{array}{ll}0 & 0 \\ 2 & 1\end{array}\right]=$ $\left[\begin{array}{cc}2 & 2 \\ 2 h^{\beta} & 1+h^{\beta}\end{array}\right]$. This implies that

$$
\operatorname{det}\left[\begin{array}{l}
C \Phi(0) \\
C \Phi(1)
\end{array}\right]=1-h^{\beta} \neq 0 ;
$$

hence then system is observable in $q=1$ step.

(3) Now we take the Riemann-Liouville-type operator; then we need values of matrix function $M_{(k)}(q)$. Observe that

$$
\begin{gathered}
M_{(0,0)}(1)=\operatorname{diag}\left(\varphi_{(1,0)}, \varphi_{(0,1)}\right)(1)=\left[\begin{array}{ll}
\alpha & 0 \\
0 & \beta
\end{array}\right], \\
M_{(1,0)}(1)=\operatorname{diag}\left(\varphi_{(2,0)}, \varphi_{(1,1)}\right)(1)=I_{2}, \quad M_{(0,1)}(1)=I_{2} ;
\end{gathered}
$$

$$
\begin{gathered}
\text { then } \Phi(1)=T_{(0,0)} M_{(0,0)}(1)+T_{(1,0)} M_{(1,0)}(1)+ \\
T_{(0,1)} M_{(0,1)}(1)=\left[\begin{array}{ll}
\alpha & 0 \\
0 & \beta
\end{array}\right]+\left[\begin{array}{ll}
1 & 2 \\
2 & 1
\end{array}\right]=\left[\begin{array}{cc}
1 \alpha & 2 \\
2 & 1+\beta
\end{array}\right] . \text { And } \\
\operatorname{det}\left[\begin{array}{l}
C \Phi(0) \\
C \Phi(1)
\end{array}\right]=2,
\end{gathered}
$$

for $\alpha \neq \beta$; hence then system is observable in $q=1$ step. 


\section{Conclusions}

From our consideration it follows that we could not distinguish which model/type of the system is better according to the controllability or the observability properties. In fact it depends on the matrices inside the systems. Moreover parameters that one can include in the description of some process involve the model and the type operator with ramifications of the used model. We cannot downplay the role of the used orders and steps. However this paper does not discuss any particular process. This is rather theoretical consideration of possible properties.

\section{Conflict of Interests}

The author declares that there is no conflict of interests regarding the publication of this paper.

\section{Acknowledgments}

The author is grateful to anonymous referees for valuable suggestions and comments, which improved the quality of the paper. The project was supported by the funds of National Science Centre granted on the bases of the decision number DEC-2011/03/B/ST7/03476. The work was supported by Bialystok University of Technology Grant G/WM/3/2012.

\section{References}

[1] K. S. Miller and B. Ross, "Fractional difference calculus," in Proceedings of the International Symposium on Univalent Functions, Fractional Calculus and Their Applications, pp. 139-152, Nihon University, Kōriyama, Japan, 1989.

[2] F. M. Atici and P. W. Eloe, "A transform method in discrete fractional calculus," International Journal of Difference Equations, vol. 2, no. 2, pp. 165-176, 2007.

[3] F. M. Atici and P. W. Eloe, "Initial value problems in discrete fractional calculus," Proceedings of the American Mathematical Society, vol. 137, no. 3, pp. 981-989, 2009.

[4] T. Abdeljawad and D. Baleanu, "Fractional differences and integration by parts," Journal of Computational Analysis and Applications, vol. 13, no. 3, pp. 574-582, 2011.

[5] T. Abdeljawad, F. Jarad, and D. Baleanu, "A semigroup-like property for discrete Mittag-Leffer functions," Advances in Difference Equations, vol. 2012, article 72, 2012.

[6] N. R. O. Bastos, R. A. C. Ferreira, and D. F. M. Torres, "Necessary optimality conditions for fractional difference problems of the calculus of variations," Discrete and Continuous Dynamical Systems, vol. 29, no. 2, pp. 417-437, 2011.

[7] R. A. C. Ferreira and D. F. M. Torres, "Fractional $h$-difference equations arising from the calculus of variations," Applicable Analysis and Discrete Mathematics, vol. 5, no. 1, pp. 110-121, 2011.

[8] N. R. O. Bastos, R. A. C. Ferreira, and D. F. M. Torres, "Discretetime fractional variational problems," Signal Processing, vol. 91, no. 3, pp. 513-524, 2011.

[9] D. Mozyrska and E. Pawluszewicz, "Local controllability of nonlinear discrete-time fractional order systems," Bulletin of the Polish Academy of Sciences, vol. 61, no. 1, pp. 251-256, 2013.

[10] D. Mozyrska, E. Girejko, and M. Wyrwas, "Comparison of $h$ difference fractional operators," in Advances in the Theory and
Applications of Non-integer Order Systems, W. Mitkowski, J. Kacprzyk, and J. Baranowski, Eds., vol. 257 of Lecture Notes in Electrical Engineering, pp. 191-197, Springer, 2013.

[11] D. Mozyrska and E. Girejko, "Overview of fractional $h$ difference operators," in Operator Theory: Advances and Applications, vol. 229, pp. 253-268, Birkhäuser, 2013.

[12] D. Mozyrska, "Controllability of $h$-difference linear control systems with two fractional orders," International Journal of Systems Science, 2013.

[13] E. Pawluszewicz and D. Mozyrska, "Constrained controllability of $h$-difference linear systems with two fractional orders," in Advances in the Thoery and Applications of Non-Integer Order Systems, W. Mitkowski, J. Kacprzyk, and J. Baranowski, Eds., vol. 257 of Lecture Notes in Electrical Engineering, pp. 67-76, Springer, 2013.

[14] D. Mozyrska and E. Pawluszewicz, "Controllability of $h$ difference linear control systems with two fractional orders," in Proceedings of the 13th International Carpathian Control Conference (ICCC '12), I. Petráš, I. Podlubny, K. Kostúr, J. Kačúr, and A. Mojžišová, Eds., pp. 501-506, May 2012.

[15] I. Podlubny, Fractional Differential Equations, vol. 198, Academic Press, San Diego, Calif, USA, 1999.

[16] T. Kaczorek, "Fractional positive linear systems," Kybernetes, vol. 38, no. 7-8, pp. 1059-1078, 2009.

[17] T. Kaczorek, "Reachability of cone fractional continuous-time linear systems," International Journal of Applied Mathematics and Computer Science, vol. 19, no. 1, pp. 89-93, 2009. 


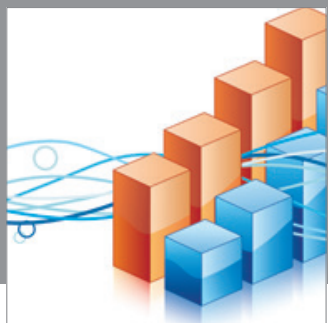

Advances in

Operations Research

mansans

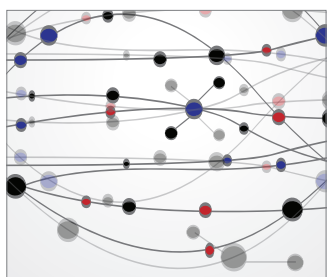

The Scientific World Journal
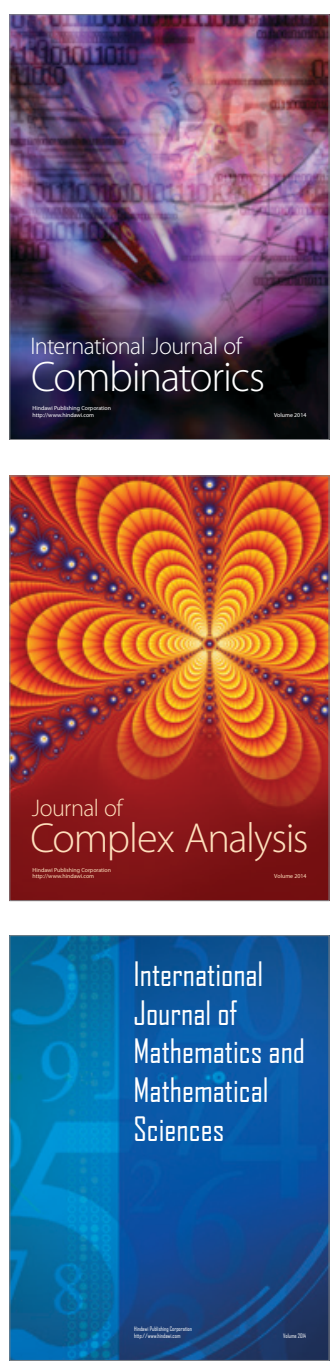
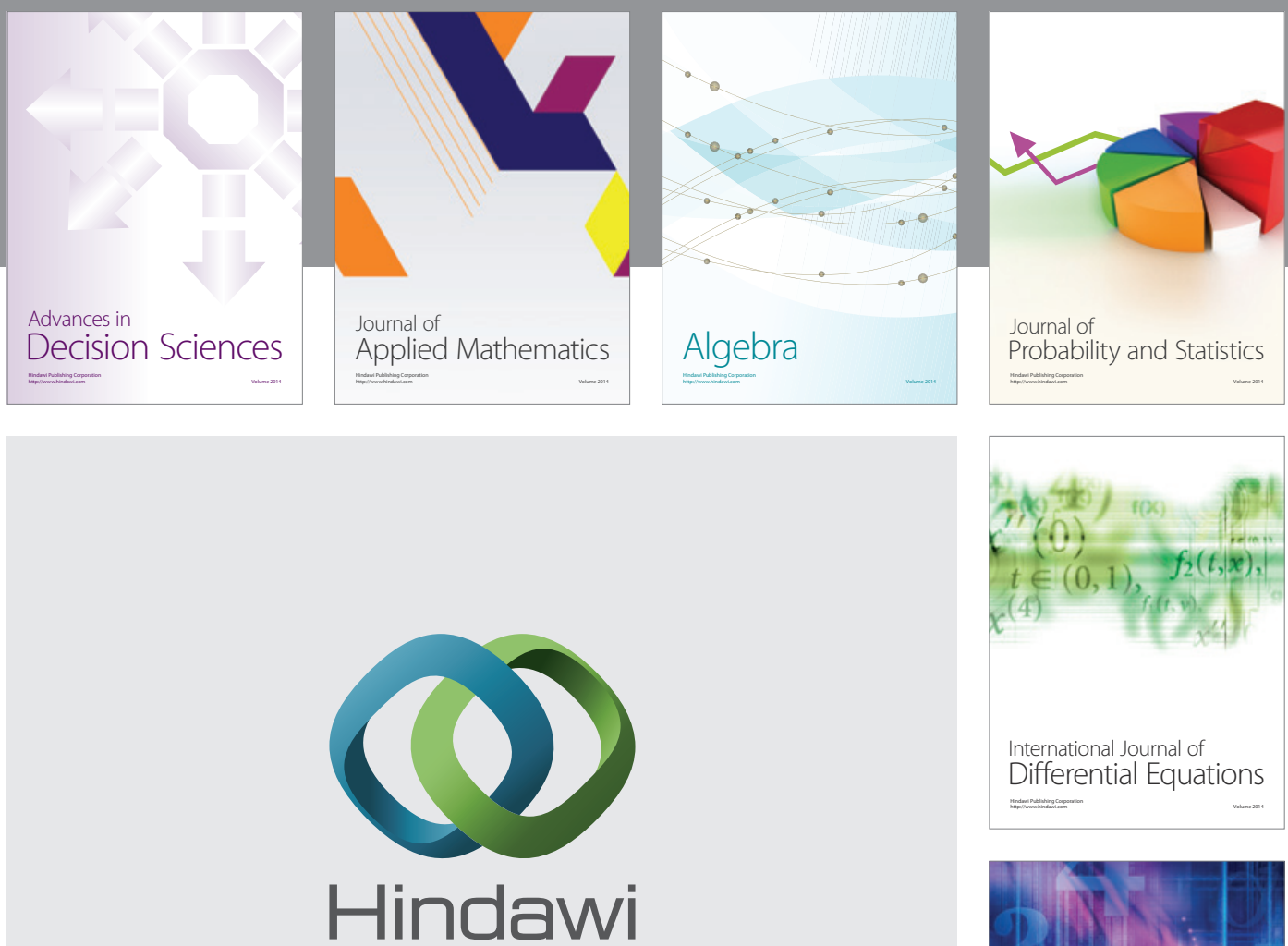

Submit your manuscripts at http://www.hindawi.com
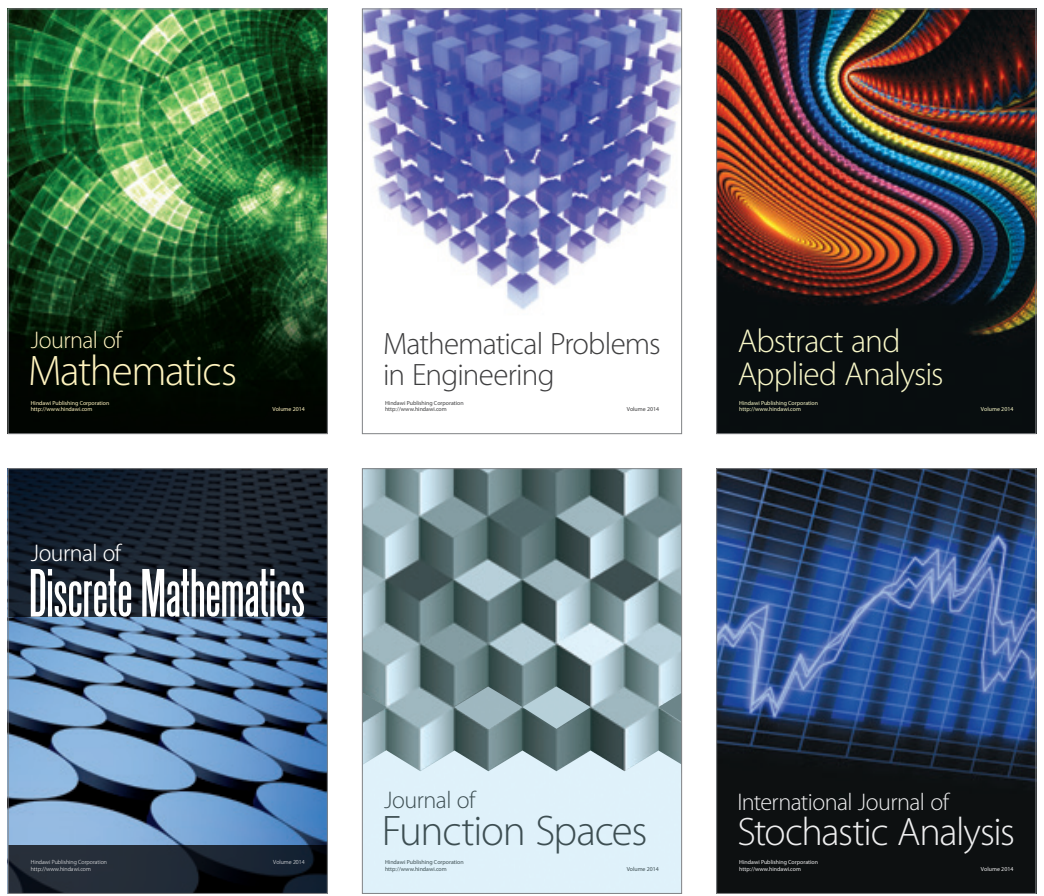

Journal of

Function Spaces

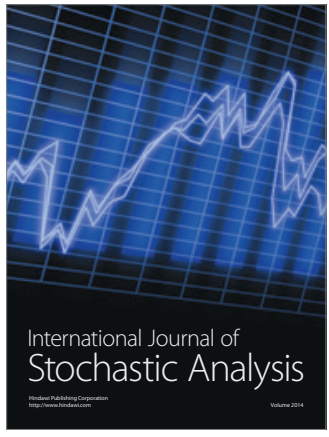

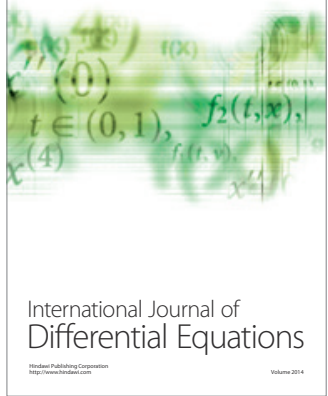
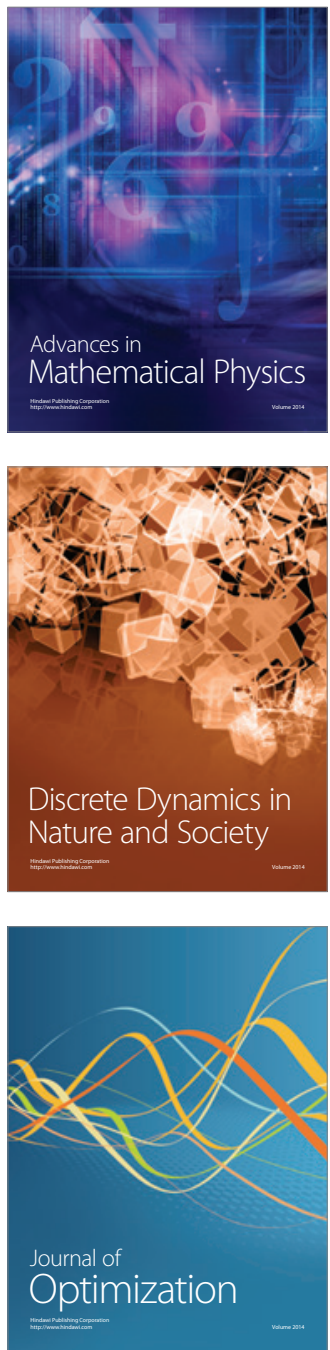